\title{
Inter-channel Stimulated Raman Scattering and its Impact in Wideband Transmission Systems
}

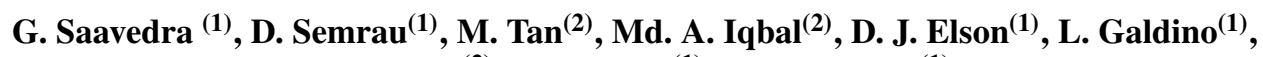 \\ P. Harper ${ }^{(2)}$, R. I. Killey ${ }^{(1)}$, and P. Bayvel ${ }^{(1)}$ \\ (1) Optical Networks Group, Dept. of Electronic \& Electrical Engineering, UCL, London WC1E 7JE, UK \\ ${ }^{(2)}$ Aston Institute of Photonic Technologies, Aston University, Birmingham B4 7ET, UK \\ uceegsa@ucl.ac.uk
}

\begin{abstract}
The impact of inter-channel stimulated Raman scattering (ISRS) in wideband optical transmission systems is studied. ISRS cross-talk due to channel modulation was found to be negligible and a good agreement was found with theoretical results.
\end{abstract}

OCIS codes: (060.4510) Optical communications; (060.4370) Nonlinear optics, fibers.

\section{Introduction}

Extending the optical bandwidth beyond the limits determined by the gain window of the currently used optical amplifiers, is a promising way to increase the capacity of optical fiber communication systems. The use of larger optical bandwidths, however, has the drawback of increasing the nonlinear effects present in the transmission system. Much attention has focused on the study of the limits on capacity imposed by Kerr nonlinearity, with a variety of models proposed to predict the performance of modern optical transmission systems [1,2]. However, as the transmitted signal powers and bandwidths are increased, stimulated Raman scattering will play an increasingly important role in limiting capacity [3]. Therefore, the impact of inter-channel stimulated Raman scattering (ISRS) needs to be addressed in the context of large bandwidth coherent optical transmission systems.

We recently developed a transmission system model, combining the inter-channel power transfer due to ISRS with the Kerr nonlinear effects described by the Gaussian noise model [4]. This model can be used to calculate wavelengthdependent SNR, optimise modulation formats and coding in the presence of ISRS and estimate wideband system capacity. One assumption of the model is that ISRS introduces negligible crosstalk due to channel modulation. The validity of this assumption is an open question. In this paper we experimentally verify this model, through measurements of the effect of ISRS in C+L band transmission systems, and found no evidence of ISRS-induced crosstalk originating from time-varying amplitudes of the interacting channels. The study is based on Nyquist shaped, polarization multiplexed, 256-ary quadrature-amplitude modulated (PM-256QAM) channels. Theoretical predictions from the model derived in [4] were verified by the experimental results.

\section{Experimental setup and methodology}

To study the effect of inter-channel stimulated Raman scattering, the experimental setup shown in Fig. 1 was used. Fourteen C-band external cavity lasers (ECLs) placed between 1529 and $1540 \mathrm{~nm}$, and spaced at $100 \mathrm{GHz}$ were

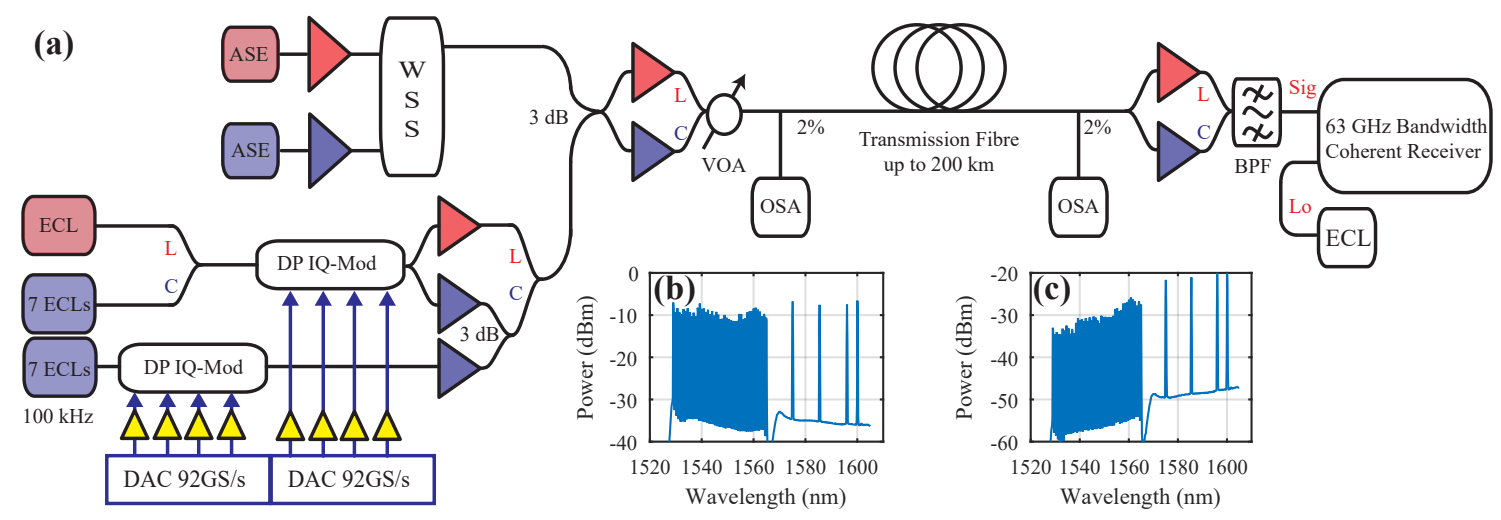

Fig. 1: (a) Experimental transmission setup. (b) and (c) correspond to the input and output spectrum at $10.7 \mathrm{dBm}$ per channel measured using $0.1 \mathrm{~nm}$ resolution. 

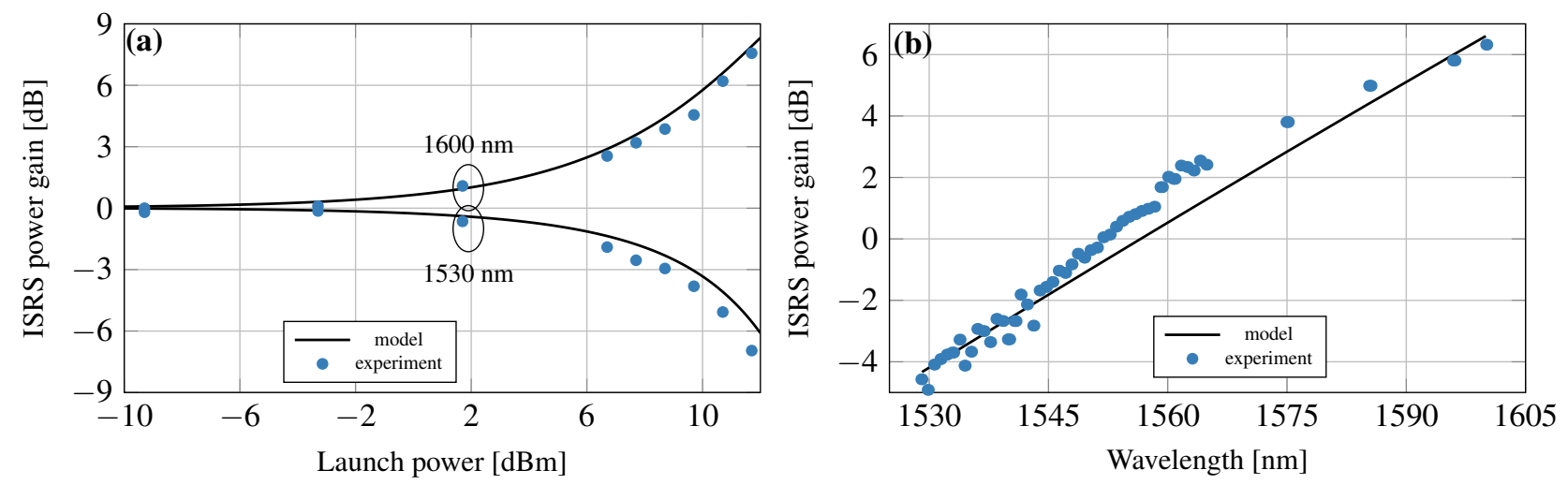

Fig. 2: (a) Power gain/loss due to ISRS experienced by the 1530 and $1600 \mathrm{~nm}$ channels as a function of launch power. (b) Power gain/loss due to ISRS as a function of the transmitted wavelength at $10.7 \mathrm{dBm}$ per channel.

connected to two dual polarization IQ modulators generating decorrelated odd and even channels. An additional Lband ECL was coupled before modulation. The electrically amplified output from the $92 \mathrm{GS} / \mathrm{s}$ digital-to-analogue converters (DACs) was used to drive the IQ-modulators. The drive signals were generated off-line and correspond to a 32 GBd 256QAM signal, shaped using a root raised cosine filter with a 1\% roll-off factor. Spectrally-shaped, amplified spontaneous emission (SS-ASE) noise was used to emulate additional interfering channels [5]. The SS-ASE was shaped as channels with $32 \mathrm{GHz}$ bandwidth and $100 \mathrm{GHz}$ spacing in the C-band, and $1000 \mathrm{GHz}$ spacing in the L-band (fewer channels were transmitted in the L-band due to the amplifier output power limitations). The modulated signals and SS-ASE were coupled together and amplified before launching into the transmission fibre.

For transmission a single fibre span allowing the effect of ISRS to be isolated and more accurately measured. Three different fibre lengths of 100,160 and $200 \mathrm{~km}$ were used, with attenuation coefficient of $0.18 \mathrm{~dB} / \mathrm{km}$, dispersion parameter of $16 \mathrm{ps} / \mathrm{nm} / \mathrm{km}$ and nonlinear coefficient of $1.31 / \mathrm{W} / \mathrm{km}$. The measured normalized Raman gain coefficient was $0.421 / \mathrm{W} / \mathrm{km}$ at a Stokes shift of $11.5 \mathrm{THz}$. The spectra of the input and output signals are shown in insets (b) and (c) of Fig. 1 respectively. After the fibre span the signals were amplified and a band-pass filter selected the channel of interest. The receiver comprised an ECL used as local oscillator, and detection was carried out with a digital coherent receiver followed by digital signal processing as detailed in [6].

In this work, signal-to-noise ratio (SNR) was used as the performance metric, and it was estimated for experimental data as the ratio between the variance of the transmitted symbols, and the variance of the noise, as in [6]. The experimental results were compared to theoretical predictions based on the model described in [4]. The model treats ISRS as a pure signal gain or loss component. This assumes that cross-talk as a result of time-varying power fluctuations of the modulated channels average out due to the high number of uncorrelated interacting channels [7]. As in [4], an effective attenuation coefficient was used in the calculation of the nonlinear interference coefficient of the channel of interest that reflects the power dependent effective length of that channel in the presence of ISRS. The average ISRS power transfer was calculated by numerically solving the Raman gain equations as described in [8] using the same input spectrum seen in inset Fig. 1 (b).

\section{Results and discussion}

The performance of two channels at different wavelengths was evaluated. The channels under test were placed at opposite ends of the transmitted spectrum, at 1530.5 and $1600 \mathrm{~nm}$. The ISRS power transfer as a function of launch power is shown in Fig. 2 (a). As the signal power was increased, ISRS depleted power from the $1530.5 \mathrm{~nm}$ channel, while at the same time amplified the channel power at $1600 \mathrm{~nm}$. ISRS resulted in a power transfer exceeding $0.2 \mathrm{~dB}$ for launch powers larger than $-5 \mathrm{dBm}$. Additionally, the power transfer as a function of wavelength is shown in Fig. 2 (b) for a fixed launch power of $10.7 \mathrm{dBm}$ per channel. In both cases the theory shows good agreement with the experimental results.

The SNR was measured as a function of launch power and is shown in Fig. 3, for the 1530 and $1600 \mathrm{~nm}$ channel in (a) and (b), respectively. In order to investigate the impact of ISRS, both channels were first transmitted only using their respective transmission band (C- or L-band), shown in red. Subsequently, a second transmission was performed using the entire $\mathrm{C}+\mathrm{L}$ band signal, shown in blue. At powers below the optimum, ISRS reduced the SNR at $1530 \mathrm{~nm}$, while it increased the SNR at $1600 \mathrm{~nm}$ due to the power transfer experienced by both channels. The gain in SNR 

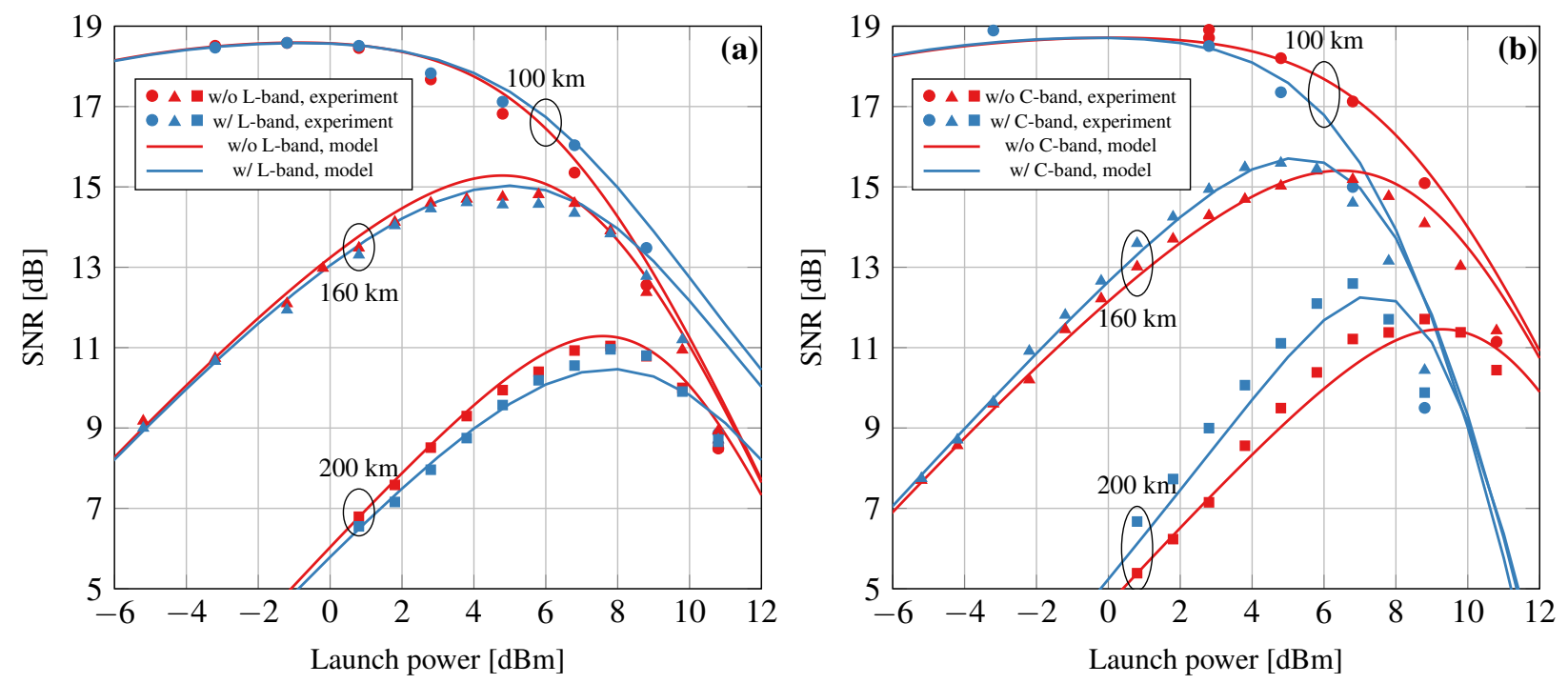

Fig. 3: SNR as a function of launch power at (a) $1530 \mathrm{~nm}$ and (b) $1600 \mathrm{~nm}$. Markers show experimental data and solid lines represent the theoretical model. The transmission of the entire spectrum $(9 \mathrm{THz})$ is shown in blue and the transmission of either only C-band or L-band channels are shown in red color.

almost exactly matches the power gain due to ISRS (Fig. 2). By way of example, the gain in SNR at $3.8 \mathrm{dBm}$ launch power is $1.5 \mathrm{~dB}$ for the $1600 \mathrm{~nm}$ channel after $200 \mathrm{~km}$ transmission. The $1.52 \mathrm{~dB}$ ISRS power gain at this launch power and distance, taken from Fig. 2(a), is almost the same. It can be concluded that ISRS cross-talk is negligible and ISRS manifests itself as a pure gain or loss component. This experimentally confirms the theoretical results in [7] and for modelling purposes this cross-talk component can be neglected, as in our model [4]. On the other hand, ISRS impacts the distortion arising from Kerr nonlinearity, as it changes the power evolution of each channel. The nonlinear distortion is decreased for low wavelength channels, where power is depleted, and increased for high wavelength channels, where amplification occurs. After $200 \mathrm{~km}$ and L-band only transmission, a maximum SNR of $11.7 \mathrm{~dB}$ was found at $8.9 \mathrm{dBm}$ per channel, however a reduction of $2 \mathrm{~dB}$ is observed when the entire C+L-band signal is transmitted due to ISRS. The effect increases as the ISRS power transfer grows.

For both wavelengths under study, the used model was found to be capable of predicting the behaviour of the system in the presence of ISRS, and shows good agreement with the experimental data for all evaluated distances.

\section{Conclusion}

The impact of inter-channel stimulated Raman scattering was studied for optical communication systems using the C and $\mathrm{L}$ band, and its impact on performance was quantified. The experimental results provide evidence for the validity of the model derived in [4], and suggest that the cross-talk effect introduced by ISRS can be neglected for modelling purposes. For higher wavelengths ISRS offers gains in the maximum SNR, and a corresponding reduction for shorter wavelengths. Optical communication systems will require solutions to minimise the reduction of information rates due to ISRS in order to overcome the capacity limits.

This is supported by Becas Chile, UK EPSRC UNLOC Programme Grant and FP7 ITN programme ICONE (608099). The authors would like to thank Oclaro for the High Bandwidth Polarization Multiplexed Quad Mach-Zehnder Modulator.

\section{References}

1. P. Poggiolini et al., J. Lightw. Technol. 32(4), 694-721 (2014)

2. A. Mecozzi et al., J. Lightw. Technol. 30(12), 2011-2024 (2012).

3. A. R. Chraplyvy, J. Lightw. Technol. 8(2), 1548-1557 (1990)

4. D. Semrau et al., Opt. Express 25(12), 13024-13034 (2017)

5. D. J. Elson et al., Opt. Express 25(16), 19529-19537 (2017)

6. G. Saavedra et al., to appear J. Lightw. Technol, doi: 10.1109/JLT.2017.2760138

7. K. Ho, J. Lightw. Technol. 18(7), 915-921 (2000)

8. S. Tariq et al., J. Lightw. Technol. 11(12), 1914-1924 (1993) 